Europhysics Letters

PREPRINT

\title{
Enhanced Quantum Reflection of Matter-Wave Solitons
}

\author{
C. LeE ${ }^{1,2}$ and J. BRAND ${ }^{1}$ \\ 1 Max Planck Institute for the Physics of Complex Systems, Nöthnitzer Straße 38, 01187 \\ Dresden, Germany \\ 2 Nonlinear Physics Centre and ARC Centre of Excellence for Quantum-Atom Optics, \\ Research School of Physical Sciences and Engineering, Australian National University, \\ Canberra ACT 0200, Australia
}

\begin{abstract}
PACS. 03.75.Lm - Tunneling, Josephson effect, Bose-Einstein condensates in periodic potentials, solitons, vortices and topological excitations.

PACS. 03.75.Mn - Multicomponent condensates; spinor condensates

PACS. 03.65.-w - Quantum mechanics.
\end{abstract}

\begin{abstract}
Matter-wave bright solitons are predicted to reflect from a purely attractive potential well although they are macroscopic objects with classical particle-like properties. The non-classical reflection occurs at small velocities and a pronounced switching to almost perfect transmission above a critical velocity is found, caused by nonlinear mean-field interactions. Full numerical results from the nonlinear Schrödinger equation are complimented by a two-mode variational calculation to explain the predicted effect, which can be used for velocity filtering of solitons. The experimental realization with laser-induced potentials or two-component BoseEinstein condensates is suggested.
\end{abstract}

In the framework of classical mechanics, a moving object will never turn back until it reaches a turning point, at which the radial velocity vanishes (the kinetic energy vanishes for one dimensional systems). At the microscopic scale, where the wave character of particles becomes important, quantum mechanics allows the reflection of a particle in a classically allowed region even when there is no classical turning point. Quantum reflection can occur above a repulsive potential barrier or an attractive potential well, and may take place in an attractive potential tail [1] or at a potential step [2]. The quantum reflection of cold atoms by a solid surface has triggered great interest both for the fundamental understanding of the implications of quantum mechanics and for potential applications of mirrors in atom optics [3]. Recently, Pasquini et al. reported the experimental observation of the quantum reflection of atoms from a dilute Bose-Einstein condensate (BEC) with up to 20 and $50 \%$ efficiency [4].

Matter-wave bright solitons are macroscopic quantum objects that may act as classical particle-like objects maintaining their integrity during collisions or while subjected to external forces. They have been prepared as self-bound droplets of atomic BECs with negative swave scattering lengths in quasi-one-dimensional waveguides [5,6]. Previously we considered conditions for the creation of soliton trains [7] and their propagation in harmonic traps [8]. In this Letter we show that a matter-wave soliton approaching an attractive potential well may experience non-classical reflection. In contrast to the finite probabilities of quantum

(c) EDP Sciences 

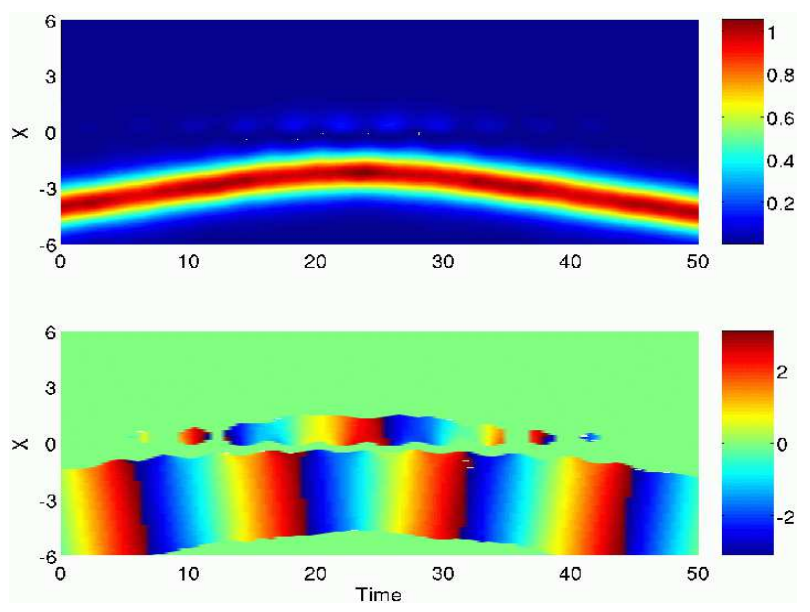

Fig. 1 - Quantum reflection of a soliton incident on an attractive potential well centred at $x=0$. The initial conditions are $A=1, x_{0}=-4$ and $v=0.1$. The upper panel shows the density and the lower one the phase evolution.

reflection of single atoms, the whole soliton reflects with very little radiative loss as seen in Fig. 1. leading to a significant enhancement of reflection due to nonlinear interactions and macroscopic coherence. Above a critical velocity we observe a sharp transition to almost complete transmission while trapping is also possible in different parameter regimes.

Solitons behave as classical particles according to perturbation theory when they move in weak external potentials [9] or by virtue of Ehrenfest's theorem in semiclassical conditions where the potential varies slowly on the size scale of the soliton. Non-classical soliton scattering is expected when the kinetic energy dominates over the soliton binding energy or in specific resonant scattering scenarios and was previously discussed for step-like [10] and square potentials [11] and impurities [12-14].

The effect of pronounced switching from quantum reflection to transmission reported in this Letter fits into neither of these categories as adiabatic conditions are broken by a strongly localised potential well but nonlinearity dominates over kinetic energy. Thus the soliton may remain intact. In the following we will discuss the various parameter regimes in detail. We report results of numerical simulations and study a two-mode variational model, which gives valuable insights into the underlying mechanism. We furthermore discuss possible experimental realizations with optical dipole potentials and incoherent solitons in two-component BECs and applications in velocity filtering.

Consider a matter-wave soliton in a one-dimensional waveguide trap approaching the centre of a localised attractive potential well $V(x)$. The dynamics is described well by the GrossPitaevskii equation (GPE), which we consider in the one-dimensional approximation [15] and write in dimensionless units as

$$
i \frac{\partial}{\partial t} \Psi(x, t)=\left[-\frac{1}{2} \frac{\partial^{2}}{\partial x^{2}}-g|\Psi(x, t)|^{2}+V(x)\right] \Psi(x, t) .
$$

The parameter $g$ is proportional to the s-wave scattering length. Without loss of generality, we assume that $g=0$ and 1 correspond to the linear and nonlinear system, respectively.

For the linear system $(g=0)$ with an attractive sech-squared-shape potential $V(x)=$ $-U \operatorname{sech}^{2}(\alpha x)$, known as Rosen-Morse potential, the quantum mechanical reflection probability 
$R$ is known exactly [16],

$$
R=\frac{\cos ^{2}\left[\frac{\pi}{2} \sqrt{1+8 U / \alpha^{2}}\right]}{\sinh ^{2}(\pi v / \alpha)+\cos ^{2}\left[\frac{\pi}{2} \sqrt{1+8 U / \alpha^{2}}\right]},
$$

and is determined by both the incident velocity $v$, which in our units coincides with the particle momentum, and the system parameters. Particles with large velocities are transmitted and particles with small velocities reflected unless the cosine term in the numerator of Eq. (2) vanishes for specific values of $U / \alpha^{2}$. In these special cases the transmission of linear waves is reflectionless. Reflection is maximised when the cosine term becomes unity. In this case, the reflection probability $R$ is greater than $50 \%$ for small velocities with $v<v_{R}=0.28 \alpha$.

For the nonlinear system $(g=1)$ with the same potential, the system supports travelling soliton solutions in the absence of the external potential $V(x)$ :

$$
\Psi=A \operatorname{sech}\left(A\left[x-x_{0}-v t\right]\right) e^{i(v x-\omega t)},
$$

where $v$ is the velocity, $A$ is the amplitude, and $x_{0}$ is the initial position. The soliton frequency $\omega=v^{2} / 2+\mu$ can be interpreted as the sum of the kinetic energy per particle and the binding energy $\mu=-A^{2} / 2$. We now consider the scattering of solitons on the potential well $V(x)$ and find that the situation may change dramatically compared to the linear case, depending on the relevant scales of the system.

In order to avoid the classical Ehrenfest regime, the length scale of the potential well $l_{V}=1 / \alpha$ should be smaller than the soliton length scale $l_{S}=1 / A[9]$. A regime of linear wave scattering can be expected when the time scale of interaction of the soliton with a localised defect is shorter than a characteristic time scale of the nonlinearity as suggested in Ref. [14] for resonant interactions. For non-resonant interactions we may rewrite this as a condition for the velocity, which should be larger than the velocity $v_{\text {disp }}=4|\mu| / l_{S}=2 A^{3}$ characterising the dispersion of a linear wave packet with the soliton parameters. In this regime we find a splitting of the soliton into reflected and transmitted portions. Finally we should compare the energy scale of the soliton $\omega$ with the energy of the lowest bound state of the potential $V(x)$ in the linear Schrödinger equation, which is [16] $E_{0}=-\left(\alpha^{2} / 2\right)\left(\sqrt{2 U / \alpha^{2}+1 / 4}-1 / 2\right)^{2}$. Following an argument of Ref. [13], we may expect a regime of strong soliton-defect interaction if $\omega \leq E_{0}$ because an overlapping between the incoming soliton and the localised bound state may lead to a resonant population transfer from the soliton into the bound state. In this regime, we find indeed that solitons at small velocities are trapped by the potential well.

What are the possibilities to meet the required conditions by choosing appropriate parameters for the soliton (31) and the potential well? From the condition for the length scales for non-Ehrenfest scattering $l_{V}<l_{S}$ we obtain $A<\alpha$. In order to find a sharp transition between reflection and transmission, the velocity scale for quantum reflection $v_{R}$ should be less than $v_{\text {disp }}$, which yields $0.28 \alpha<3 A^{3}$. Both conditions are compatible but require both $\alpha$ and $A$ to be larger than roughly $1 / 3$. Finally, strong defect interactions can be avoided when $\mu>E_{0}$. We find $A^{2}<\alpha^{2}\left(\sqrt{2 U / \alpha^{2}+1 / 4}-1 / 2\right)$, which can always be satisfied if $U$ is chosen large enough.

We now consider an initially well separated soliton with $A=1$ approaching a potential well with $U=4$ and $\alpha=2$. Figure 1 shows the result of a numerical integration of the GPE (11). The relevant scales are $l_{S}=1, l_{V}=1 / 2, v_{\text {disp }}=2, \mu=-1 / 2$, and $E_{0}=-4$. The time evolution shown in Fig. 1 indicates that the matter-wave soliton can indeed be reflected by the attractive potential. An important observation from Fig. 1 is that the potential well is populated transiently by a small portion of the matter wave, with a phase difference of $\pi$ compared to the soliton. 


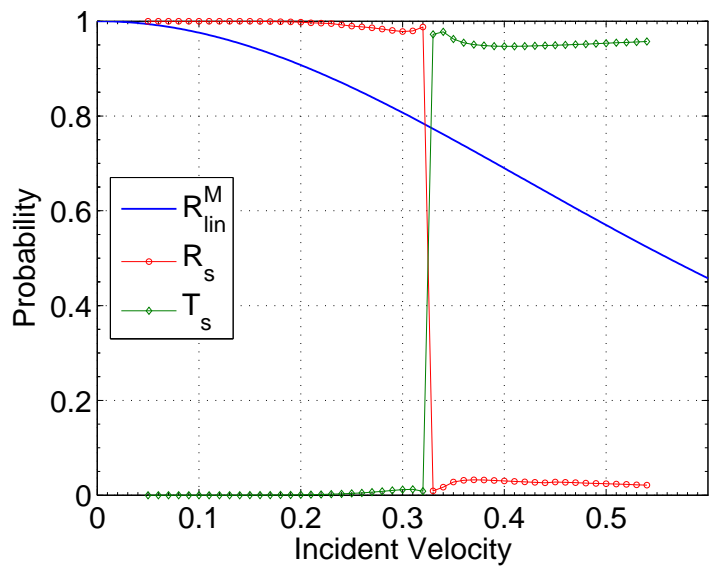

Fig. 2 - Reflection $R$ and transmission $T$ versus incident velocity $v$ for $U=4$ and $\alpha=2$. The $R_{\text {lin }}^{M}$ is the maximum possible reflection in the linear system $(g=0)$ obtained by modifying the potential depth. The $R_{s}$ and $T_{s}$ corresponds to the nonlinear system supporting an initial soliton with $A=1$ at $x_{0}=-10$.

Due to the mean-field interaction, the dependence of the reflection probability on the incident velocity is dramatically changed. In comparison with the smooth transition between reflection and transmission in linear systems, the nonlinear interaction makes the transition much sharper with a well-defined critical velocity, seen in Fig. 2 . Here, we calculated $R_{s}$ and $T_{s}$ as the reflected and transmitted portion of atoms by integrating the density $|\Psi(x, t)|^{2}$ after the collision at $t=50$ on the left side and right side of the well, respectively.

In addition to reflected and transmitted waves, some atoms can be trapped in the potential well. This process is particularly important for small depths of the attractive potential well due to resonant energy transfer [13], but can be mostly avoided if the potential well is deep enough. Besides the travelling soliton and the trapped mode, there are small amounts of radiation, in particular when the incident velocity is in the critical region between reflection and transmission. The soliton scattering problem as as shown in the lower panel of Fig. 2 is dominated by reflection when the incident velocity is below 0.32 but is dominated by transmission when the incident velocity is above 0.33. In these two dominant regimes, at least $96 \%$ of particles are reflected or transmitted. We have varied the depth of the potential well $U$ and found that the crossover velocity $v_{R}=0.28 \alpha$ (obtained from $R_{\text {lin }}^{M}=1 / 2$ ) of the linear system is a reasonable estimate for the critical velocity for the quantum reflection of solitons. However, while the actual reflection $R$ of Eq. (2) for the linear system shows a very sensitive dependence on $U$, we find no such strong dependence for the scattering of solitons. The cases of reflectionless scattering that occur in the linear problem for specific values of $U=\alpha^{2}\left[(2 N+1)^{2}-1\right] / 8$ (where $N$ is a non-negative integer) can be related to resonances at zero scattering energy [17]. In the nonlinear case these resonances do not survive because phase relationships are modified by nonlinear interactions [14]. For potentials with reflectionless scattering $R(v) \equiv 0$ of linear waves, the soliton still shows a reflection characteristic as in Fig. 2. and thus presents an extreme enhancement of the quantum reflection probability from exactly zero to more than 0.96 .

The quantum reflection of the incident soliton can be interpreted and analysed within a 
simple two-mode picture. To this end we write

$$
\Psi(x, t)=\Psi_{S}(x, t)+\Psi_{T}(x, t),
$$

where $\Psi_{S}(x, t)$ represents the soliton and $\Psi_{T}(x, t)$ is an immobile trapped mode which is of importance if the potential is sufficiently deep and localised to escape the perturbative regime. When the two modes overlap, the off-diagonal terms of the attractive potential, $\Psi_{S}^{*}(x, t) V(x) \Psi_{T}(x, t)$ and $\Psi_{T}^{*}(x, t) V(x) \Psi_{S}(x, t)$, will result in a repulsive force between two modes when they are out of phase. Additionally, similar to the interaction between two coherent solitons [18], the mean-field interaction will induce a repulsive (or attractive) force between two modes when they are out of phase (or in phase). When the soliton moves close to the potential centre, the soliton will be reflected if the repulsion overcomes the attractive effects.

A collective coordinate approach has been used in Ref. [13] to study the interactions of the soliton and the defect mode for the case of a delta function defect potential $V_{D}(x)=-A_{P} \delta(x)$. The delta defect with $A_{P} \sim 2 U / \alpha$ is a reasonable approximation for a strongly localised potential well as long as $l_{V} \ll l_{S}$ and thus we may expect to obtain some insight from the model of Ref. [13] for our case. The variational ansatz for the trapped mode is the parametrised solution of the nonlinear localised state with the potential $V_{D}(x)$ and is written as $\Psi_{T}=A_{T} \operatorname{sech}\left[A_{T}|x|+\operatorname{arctanh}\left(\frac{A_{P}}{A_{T}}\right)\right] \exp \left(i \phi_{T}\right)$. Here, the trapped mode amplitude $A_{T}$ and phase $\phi_{T}$ appear as time-dependent variational parameters. For the soliton mode we choose $\Psi_{S}=A_{S} \operatorname{sech}\left[A_{S} x-Q\right] \exp \left(i V x+i \phi_{S}\right)$, where the position $Q$, velocity $V$, amplitude $A_{S}$, and phase $\phi_{S}$ are variational parameters. The Lagrangian for the collective coordinates is derived from the standard variational formulation of the GPE (11) [15] and simplified by neglecting interaction terms not proportional to $V(x)$ [13] to yield

$$
\begin{aligned}
L= & -2 A_{S} \frac{d \phi_{S}}{d t}-2 Q \frac{d V}{d t}-2\left(A_{T}-A_{P}\right) \frac{d \phi_{T}}{d t}+\frac{1}{3} A_{S}^{3}-A_{S} V^{2}+\frac{1}{3} A_{T}^{3}-\frac{2}{3} A_{P}^{3} \\
& +A_{P} A_{S}^{2} \operatorname{sech}^{2} Q+2 A_{P} A_{S} \sqrt{A_{T}^{2}-A_{P}^{2}} \operatorname{sech} Q \cos \left(\phi_{T}-\phi_{S}\right) .
\end{aligned}
$$

The equations of motions for the collective coordinates $Q, V, A_{S}, \phi_{S}, A_{T}$, and $\phi_{T}$ are given by the Euler-Lagrange equations of $L$ and can be solved straightforwardly by numerical integration.

The numerical results as seen in Fig. 3 show that the incident soliton can indeed be reflected by the attractive potential well when the incident velocity is sufficiently small. Otherwise, when the incident velocity is larger than a certain critical value, the soliton will travel through the potential. When the distance between the soliton and the trapped mode is small enough, population transfer between them may occur. For this reason, the soliton mode obtains a time delay when it approaches the potential centre and is decelerated. The deceleration of the soliton mode becomes more and more significant with decreasing incident velocity. For sufficiently slow incident velocity, the velocity can finally change direction, and the incident soliton is reflected. This behaviour is obviously contrary to the classical case. To find where this counterintuitive behaviour comes from, we track the evolution of the relative phase between the two modes. We find that the two modes are out of phase (the relative phase oscillates around $\pi$ ) when the soliton mode approaches the potential centre. Thus the overlapping between modes induces a repulsive force between them. Clearly, these variational results are qualitatively consistent with the directly numerical results from the GPE (1). However, differences appear in quantitative results such as the critical velocity between reflection and transmission.

The sharp transition between reflection and transmission may be useful for filtering solitons according to their velocity. As a particular potential has an associated critical velocity $v_{c}$, it may discriminate between solitons with velocities below and above $v_{c}$. A sequence of potentials 

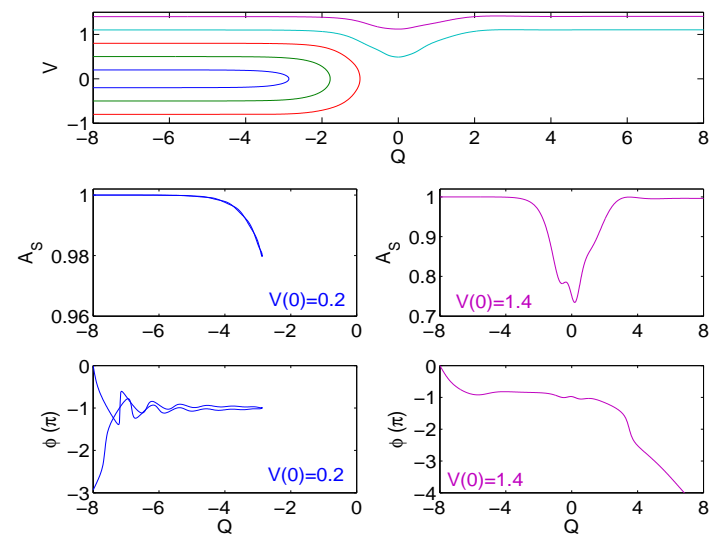

Fig. 3 - Soliton trajectories from the variational Eq. (5) are shown for $A_{P}=2.0$ and initial conditions $A_{S}(0)=1.0$ and $Q(0)=-8.0$. The upper panel shows the velocity $V$ and position $Q$ for different incident velocities $V(0)$. Below, the amplitude $A_{S}$ and relative phase $\phi=\phi_{T}-\phi_{S}$ are shown for the particular trajectories with $V(0)=0.2$ (reflected, left panels) and $V(0)=1.4$ (transmitted, right panels).

with different critical velocities may be used to specifically select solitons with velocities in a given interval. In conjunction with a potential ramp where solitons are accelerated with $\ddot{Q}=-M V^{\prime}(Q)$ it should also be possible to select solitons according to their mass $M$.

The specific choice of the scattering potential $V(x)$ as a Rosen-Morse potential is not essential for the observed effects and was made for convenient comparison of the soliton scattering to the analytically known reflection probability of the linear system. We have varied the shape of the potential well in numerical simulations in order to study its influences on the nonlinear scattering properties. In the case of a Gaussian potential well, the picture remains qualitatively the same with very little variation compared to a Rosen-Morse potential with the same width and depth. In the case of rectangular potential shape the situation changes and instead of a sharp transition between almost complete reflection and transmission we find an interval of velocities where the transition occurs. Within the transition interval the soliton is typically split into two wave packets which propagate as solitons in addition to some smallamplitude radiation. A qualitative explanation may be that wave diffraction on the sharp edges of the well effectively reduces the coherence required for cohesion of the soliton. The details of these studies will be published elsewhere [21].

In order to observe the quantum reflection of solitons induced by a finite potential well, one can use a tightly focussed red-detuned laser beam to form an attractive Gaussian shape potential. In order to see quantum reflection, the laser focus has to be brought down to the order of the soliton length scale, which was $l_{S} \approx 1.7 \mu \mathrm{m}$ in the experiment of Ref. [6]. A different possibility could be realized by another, heavy and incoherent soliton. Such could be realized with a two-component atomic BEC [19] with all negative $s$-wave lengths, which can be adjusted by using Feshbach resonances [20]. In a quasi one-dimensional external trap, the system supports well-separated incoherent solitons. When the difference between the soliton amplitudes controlled by the atomic numbers is very large, the soliton with large atomic number is almost unchanged when they approach each other. In the limit of only one atom in the small component, the intra-component mean-field interaction in the small component 
is absent and the system is equivalent to the linear case of Eq. (1). Otherwise, we have a wider soliton with small atom number moving in an attractive potential well of RosenMorse type formed by the narrower soliton with a large number of atoms. For this case, our numerical results show that quantum reflection of the wider soliton does occur for sufficiently slow relative velocity [21].

In summary, we predict that quantum reflection can occur to a kind of macroscopic quantum objects, atomic matter-wave bright solitons. The pronounced switching between reflection and transmission is a characteristic behaviour that should be observable for sufficiently well localised and deep potential wells, such as created by a strongly focussed red-detuned laser beam or a second, incoherent soliton. Possible applications lie in sensitive velocity and mass filtering for nonlinear atom optics but may extend into other areas like soliton-based optical computing. The enhanced switching behaviour is induced by the nonlinear mean-field interaction and will also occur in the interaction with other types of potentials. Interesting further questions like the possibility of quantum reflection of matter-wave solitons from solid surfaces lie outside the scope of this Letter.

We acknowledge useful comments by S. FLACH, and Y. S. KIVShaR,. This work is partially supported by the Australian Research Council (ARC).

\section{REFERENCES}

[1] Friedrich H. and Trost J., Phys. Rep., 397 (2004) 359.

[2] Cote R., Friedrich H. and Trost J., Phys. Rev. A, 56 (1997) 1781.

[3] Friedrich H. and Jurisch A., Phys. Rev. Lett., 92 (2004) 103202; Druzhinina V. and DeKieviet M., Phys. Rev. Lett., 91 (2003) 193202; Cote R. and Segev B., Phys. Rev. A, 67 (2003) 041604(R); F. Shimizu, Phys. Rev. Lett., 86 (2001) 987.

[4] Pasquini T. A., et Al., Phys. Rev. Lett., 93 (2004) 223201.

[5] Strecker K. E., ET AL., Nature, 417 (2002) 150.

[6] Khaykovich L., et AL., Science, 296 (2002) 1290.

[7] Carr L. D. and Brand J., Phys. Rev. Lett., 92 (2004) 040401; Phys. Rev. A, 70 (2004) 033607.

[8] Hai W., Lee C., and Chong G., Phys. Rev. A, 70 (2004) 053621.

[9] Kivshar Y. S. and Malomed B. A., Rev. Mod. Phys., 61 (1989) 763.

[10] Aceves A. B., et Al., Phys. Rev. A, 39 (1989) 1828; Kivshar Y. S., et Al., Phys. Rev. A, 41 (1990) 1677; Frauenkron H. and Grassberger P., Phys. Rev. E, 53 (1996) 2823.

[11] Sakaguchi H. and Tamura M., J. Phys. Soc. Jpn., 73 (2004) 503; Rapedius K., Et Al., arXiv: cond-mat/0507679 (2005).

[12] Forinash F., Peyrad M., and Malomed B., Phys. Rev. E, 49 (1994) 3400; Cao X. D. and Malomed B. A., Phys. Lett. A, 206 (1995) 177.

[13] Goodman R. H., Holmes P. J., and Weinstein M. I., Physica D, 192 (2004) 215.

[14] Miroshnichenko A. E., Flach S., and Malomed B., Chaos, 13 (2003) 874.

[15] Pitaevskit L. and Stringari S., Bose-Einstein Condensation (Clarendon, Oxford) 2003.

[16] Lamb G. L., Elements of Soliton Theory (Wiley, New York) 1980.

[17] Newton R. G., Scattering Theory of Waves and Particles (Springer-Verlag, New York) 1982.

[18] Stegeman G. I. and Segev M., Science, 286 (1999) 1518.

[19] Myatt C. J., et Al., Phys. Rev. Lett., 78 (1997) 586.

[20] Inouye S., ET AL., Nature, 392 (1998) 151.

[21] LeE C. and Brand J., to be published. 